\title{
Chloroplasts regulate leaf senescence: delayed senescence in transgenic ndhF-defective tobacco
}

\author{
JM Zapata ${ }^{1,2}$, A Guéra ${ }^{1,2}$, A Esteban-Carrasco ${ }^{1}, M_{\text {Martín }}{ }^{1}$ and \\ B Sabater ${ }^{\star 1}$ \\ 1 Departamento de Biología Vegetal, Universidad de Alcalá de Henares, Alcalá \\ de Henares, 28871-Madrid, Spain \\ 2 These authors contributed equally to this study. \\ * Corresponding author: B Sabater, Departamento de Biología Vegetal, \\ Universidad de Alcalá de Henares, Alcalá de Henares, 28871-Madrid, Spain. \\ Tel: + 3491 8854934; Fax: + 3491 8855066; \\ E-mail: bartolome.sabater@uah.es
}

Received 03.12.04; revised 16.2.05; accepted 30.3.05; published online 20.5.05 Edited by M Piacentini

\begin{abstract}
Mitochondrial involvement has not been identified in the programmed cell death (PCD) of leaf senescence which suggests that processes such as those involving reactive oxygen species (ROS) are controlled by chloroplasts. We report that transgenic tobacco $(\Delta n d h F)$, with the plastid ndhF gene knocked-out, shows low levels of the plastid Ndh complex, homologous to mitochondrial complex I, and more than a 30-day-delay in leaf senescence with respect to wt. The comparison of activities and protein levels and analyses of genetic and phenotypic traits of $w t x \Delta n d h F$ crosses indicate that regulatory roles of mitochondria in animal PCD are assumed by chloroplasts in leaf senescence. The $\mathrm{Ndh}$ complex would increase the reduction level of electron transporters and the generation of ROS. Chloroplastic control of leaf senescence provides a nonclassical model of PCD and reveals an unexpected role of the plastid $n d h$ genes that are present in most higher plants.

Cell Death and Differentiation (2005) 12, 1277-1284.

doi:10.1038/sj.cdd.4401657; published online 20 May 2005
\end{abstract}

Keywords: chloroplast; leaf senescence; $n d h$ genes; programmed cell death; reactive oxygen species

Abbreviations: MDA, malondialdehyde; PCD, programmed cell death; ROS, reactive oxygen species; SOD, superoxide dismutase

\section{Introduction}

Leaf senescence is a regulated process of programmed cell death $(P C D)^{1,2}$ coordinated with the development of other plant organs such as seeds, more apical leaves or storage structures. Apoptotic PCD processes take place during the senescence of different types of plant cells in which mitochondria integrate diverse signals. ${ }^{3-5}$ However, evidence remains elusive for the involvement of mitochondria during leaf senescence ${ }^{1}$ which suggests ${ }^{3,6}$ that the regulatory role of mitochondria in animal PCD could be assumed by chloroplasts in leaves. This possibility seems feasible because, in leaves, chloroplasts are the main source of reactive oxygen species $(\mathrm{ROS})^{3,7}$ that increase and are involved both in animal $\mathrm{PCD}^{8,9}$ and leaf senescence. ${ }^{6,7,10}$ In fact, ROS scavengers, such as superoxide dismutase (SOD), extend the life span when overexpressed in Drosophila ${ }^{11}$ whereas their induction is impaired during leaf senescence. ${ }^{12}$ The involvement of chloroplasts, instead of mitochondria, in the senescence of leaves would reveal a prominent role of ROS in PCD and the emergence of different systems ${ }^{13}$ during evolution to trigger the production of ROS.

Chloroplasts of higher plants contain the Ndh complex (one per 100-200 photosystem complexes), homologous to the NADH dehydrogenase or complex I (EC 1.6.5.3) of the mitochondrial respiratory chain, which catalyzes the reduction of plastoquinone with $\mathrm{NADH} .{ }^{14-16}$ In all, 11 genes $(n d h)$ of plastid DNA encode ${ }^{17-19}$ components of the Ndh complex which participates in a chlororespiratory electron transport chain $^{15,20,21}$ that regulates the redox state of transporters to optimize $^{22}$ the rate of cyclic electron transport. In chlororespiration, the Ndh complex provides electrons to plastoquinone, and the Mehler reaction (producing superoxide anion radical, $\mathrm{O}_{2}^{-}$, which is converted to $\mathrm{H}_{2} \mathrm{O}_{2}$ by $\mathrm{SOD}$ ) and peroxidase/oxidase reactions remove electrons from reduced iron sulfur protein and reduced plastoquinone, respectively.

The expression of $n d h$ genes and the activity of the $\mathrm{Ndh}$ complex increase during senescence. ${ }^{15,21,23-25}$ These increases are accompanied by an increase of thylakoid peroxidase but not of SOD, ${ }^{12,23}$ in contrast to the response of young leaves in which the three chlororespiratory activities increase in response to different stress situations. By increasing the level of the reduced forms of electron transporters, the increase of $\mathrm{Ndh}$ in senescent leaves must increase the rate of the Mehler reaction producing $\cdot \mathrm{O}_{2}^{-} \cdot 6,7,10$ By comparison, the release of cytochrome $c$ from mitochondria in $\mathrm{PCD}^{3,8}$ interrupts the respiratory electron transport, increasing the level of the reduced forms of electron transporters and the production of ROS.

Evidence for a role of the mitochondrial complex I during animal senescence ${ }^{26,27}$ suggests additional similarities between mitochondria-regulated PCD and chloroplast-regulated leaf senescence. However, the increase of ndh gene expression during senescence does not by itself demonstrate a regulatory role of the Ndh complex and chloroplasts in leaf senescence similar to that of mitochondria in animal PCD. In this work, we report a more definitive proof with a transgenic tobacco (containing an insertion-inactivated plastid ndhF gene), which shows delayed leaf senescence.

\section{Results}

The $\Delta n d h F$ transgenic tobacco was described previously ${ }^{20}$ and was obtained from Nicotiana tabacum, cv. Petit Havana 
(wt) by inactivating the plastid $n d h F$ gene by insertion of a cassette containing the spectinomycin resistance gene aad $A$ (Figure 1a). The $\Delta n d h F$ and $w t$ tobacco genotypes were distinguishable because the amplification with F2/F4 primers (Figure 1a) produced a $515 \mathrm{bp}$ band with wt DNA and a $1928 \mathrm{bp}$ band with $\Delta n d h F$ DNA. ${ }^{20}$ A faint 515 bp band was amplified, in addition to the stronger $1928 \mathrm{bp}$ band, with $\Delta n d h F$ tobacco DNA (Figure $1 \mathrm{~b}$ ). Also faint bands of NDH-F, $\mathrm{J}$ and $\mathrm{K}$ polypeptides and of $\mathrm{Ndh}$ activity were detected in $\Delta n d h F$ tobacco (Figure $1 \mathrm{c}$ and $\mathrm{d}$ ) indicating that it was not completely homoplastomic. Among the approximately 10000 copies of plastid DNA estimated per cell, $\Delta n d h F$ tobacco still contains a few copies of wt nontransformed plastid DNA. In any case, the level of the Ndh complex was very low in $\Delta n d h F$ tobacco in comparison with wt. PCR amplification with different primers and sequencing around cassette/ndhF joints (Figures S1 and S2 in Online Supplementary Information) confirmed that the spectinomycin cassette was inserted at the indicated positions of the plastid $n d h F$ gene in $\Delta n d h F$ tobacco.

No significant difference was detected in the growth and morphology of $w t$ and $\Delta n d h F$ before the reproductive stage of development (around 80 days after germination) (Figure 2). Usually, $\Delta n d h F$ bloomed 7 days before wt tobacco. Similarly to many plants, the flowering in wt tobacco is accompanied by the senescence (as judged by the yellowing that progresses from basal to apical leaves) of the branch supporting the reproductive structures. However, in contrast to $w t$, the leaves of $\Delta n d h F$ tobacco did not show senescence until the late stages of fruit development, around 40 days after flowering (Figure 2). The leaf senescence in $\Delta n d h F$ is delayed by more than 30 days with regard to $w t$ tobacco as plates in Figure 2 show for successive time periods after germination. The delayed senescence in $\Delta n d h F$ was also tested by the classical assay of chlorophyll loss in detached leaves incubated in water in the dark. ${ }^{28}$ Figure $3 a$ shows that, after 24 and $48 \mathrm{~h}$ incubations, wt leaf disks lost 15 and 25\% chlorophyll, respectively, while leaf disks of $\Delta n d h F$ barely lost $5 \%$ chlorophyll after $48 \mathrm{~h}$ incubations. The level and the activity of the Ndh complex increased during the incubation of leaf disks of wt but not of $\Delta n d h F$ tobacco (Figure 3b). The Ndh complex is responsible for the increase in chlorophyll fluorescence (dependent on the level of reduced plastoquinone) after the interruption of actinic illumination. ${ }^{20}$ Accordingly, fluorescence rose in wt but not $\Delta n d h F$ incubated leaf disks (Figure $3 \mathrm{c}$ ). This is another indication that the $\mathrm{Ndh}$ complex remained at a low level during incubations of $\Delta n d h F$ but not of $w t$ leaf disks. As a comparison, the activity of the chlororespiratory thylakoid peroxidase strongly increased during the incubation of leaf disks of $w t$ tobacco (Figure 3d) but to a lesser extent in $\Delta n d h F$.

One of the best characterized actions of ROS is the damage to membrane lipids, which may be estimated by the production of malondialdehyde (MDA) ${ }^{21}$ Indicative of the relative production of ROS, the levels of MDA were significantly lower in $\Delta n d h F$ (black bars) than in wt (white bars) basal leaves of 72-day-old plants (Figure 4a). At this age, no leaf yellowing was yet apparent, although the first flowers had appeared in $\Delta n d h F$ whereas no flowering was detected in wt. For a comparison at similar physiological stages, MDA was also determined in leaves of 79-day-old wt (gray bars) that by then showed the first flowers. Again, the MDA level was approximately 135 and $85 \%$ higher in the

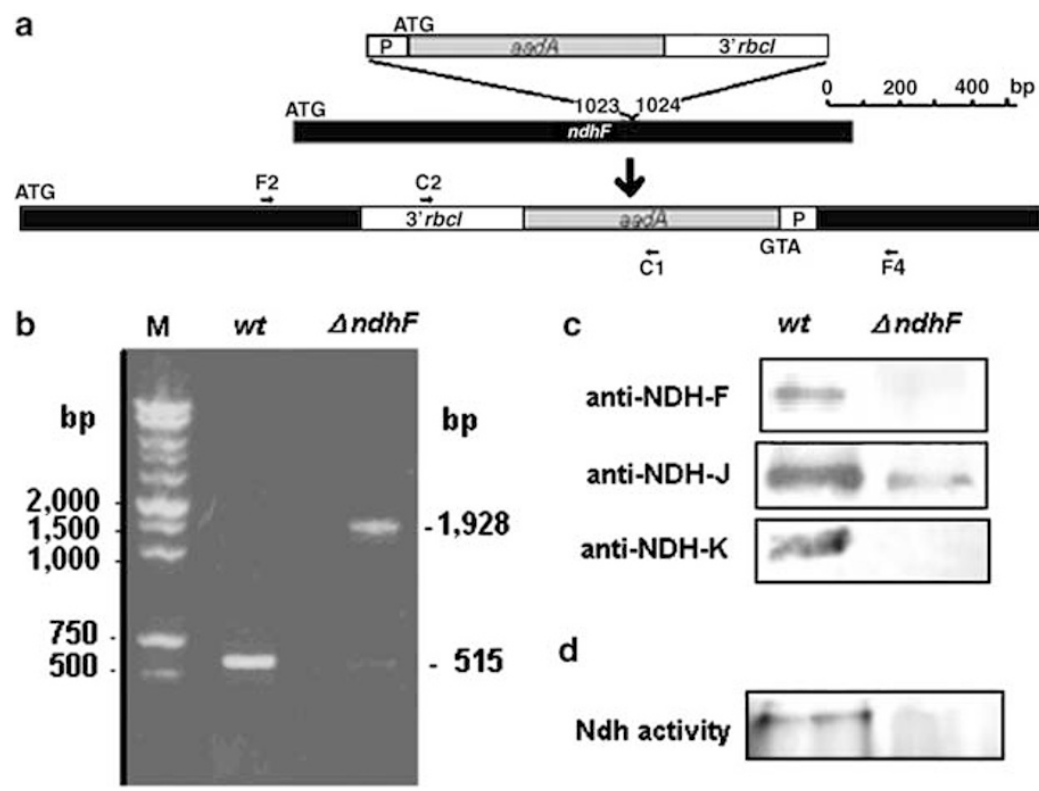

Figure 1 Insertion-inactivation of $n d h F$ gene in tobacco. (a) Map showing the antiparallel insertion of the aadA cassette in the $n d h F$ gene of $\Delta n d h F$ tobacco and the primers used to identify the genotypes. (b) PCR amplification products using primers F2/F4 and DNA isolated from the fourth leaves of wt and $\Delta$ ndhF 40 days after germination. (c) Western blot with antisera raised against NDH-F, NDH-J and NDH-K polypeptides of the Ndh complex after SDS-PAGE of $15 \mu \mathrm{g}$ protein extracts prepared from the fourth leaves of wt and $\Delta n d h F$ tobacco 40 days after germination. (d) Zymographic detection of the NADH dehydrogenase activity of the Ndh complex after native electrophoresis of $120 \mu \mathrm{g}$ protein solubilized from thylakoid preparations of the fourth leaves of wt and $\Delta$ ndhF tobacco 40 days after germination. Details of PCR amplification, the source of the specific antisera, preparation of extracts, electrophoretic and Western-blot methods and zymographic identification/determination are described in previous publications ${ }^{15,16,20,25}$ and in Online Supplementary Information 
45

\section{Days after} germination

wt
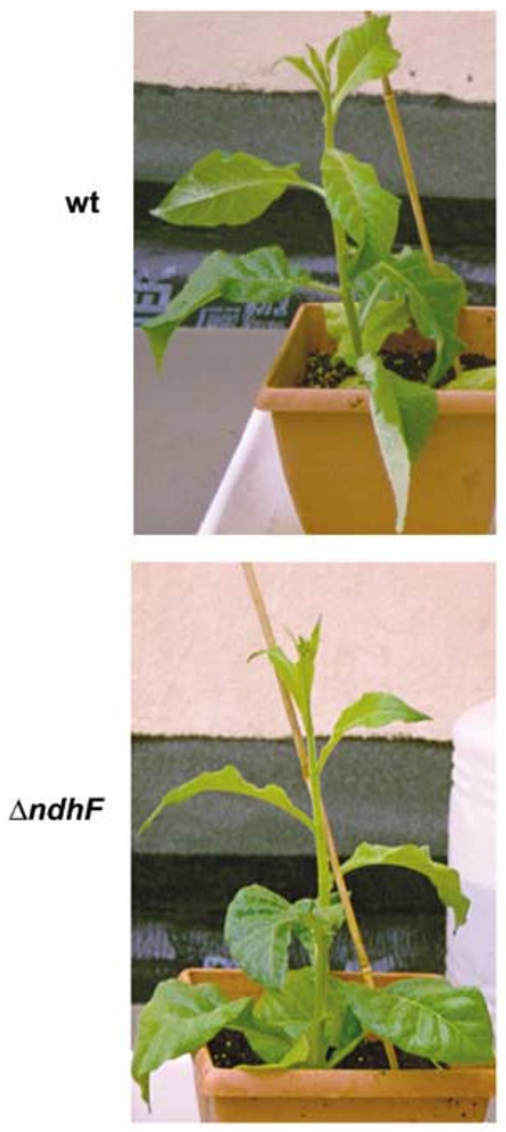

90
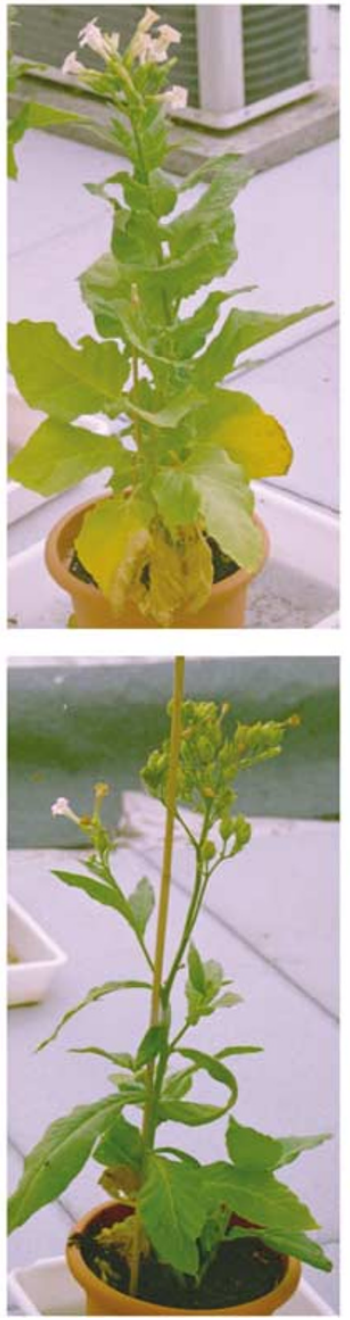

120
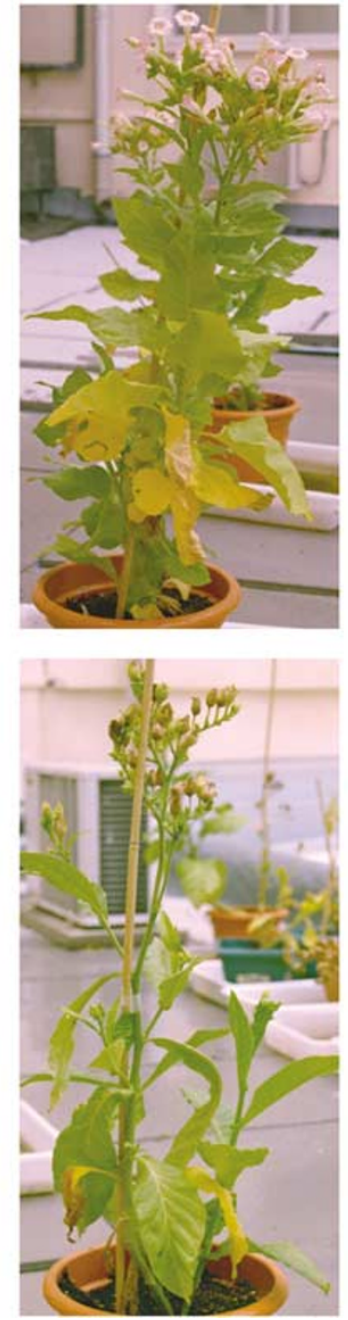

140

(glasshouse)
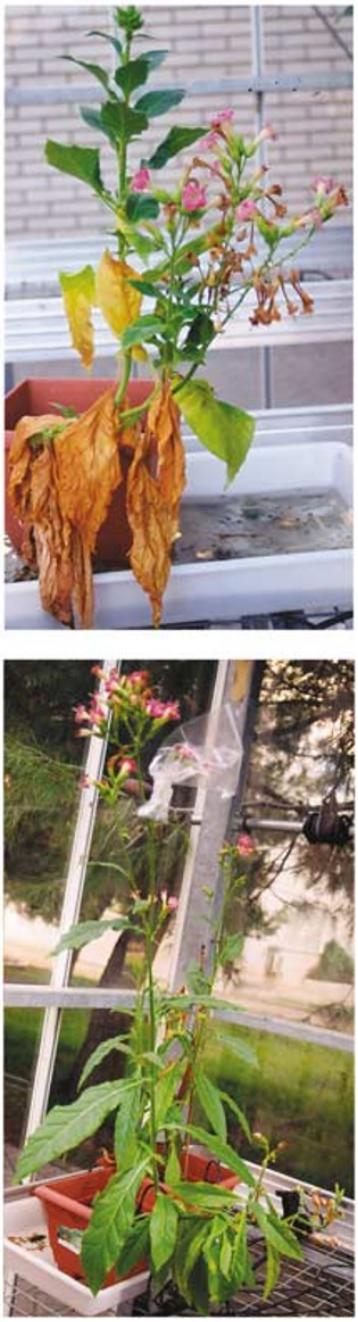

Figure 2 Leaf senescence in intact $w t$ and $\Delta n d h F$ tobacco plants. The first six plates (upper and lower) show successive stages of the development of wt and $\Delta n d h F$ tobacco plants grown simultaneously (March to July of 2003) in the open air environment for the indicated days after germination. The two plates on the right show 140day-old wt and $\Delta n d h F$ tobacco plants grown simultaneously (December 2003 to May 2004) in the glasshouse

second and fourth leaves of $\Delta n d h F$ than in the corresponding $w t$ leaves. Even the eighth leaf, that had similar MDA levels in 72-day-old $\Delta n d h F$ and $w t$, showed a small increase of MDA in 79-day-old flowering wt. The initial higher level of MDA in wt versus $\Delta n d h F$ increased slightly when leaf discs were incubated during $48 \mathrm{~h}$ in the leaf senescence assay (Figure 4b).

The delayed senescence of $\Delta n d h F$ tobacco (Figures 2 and 3 ) strongly suggested that the expression of $n d h$ genes is required for leaf senescence. To exclude possible side effects of the aadA gene delaying senescence in $\Delta n d h F$ tobacco, resistance to spectinomycin, plastid genotype and senescence phenotype were investigated in crosses of $w t$ and $\Delta n d h F$ tobacco plants. At least $50 \mathrm{~F} 1$ seeds of both

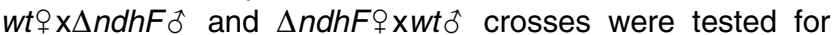
germination and development in aseptic agar cultures with or without spectinomycin. Germination percentages always ranged between 82 and $90 \%$, producing green viable seedlings when the seeds were sown in agar cultures lacking spectinomycin. However, only seeds of $\Delta n d h F+\times w t{ }^{*}$ crosses developed viable green seedlings when sown in agar cultures containing $300 \mathrm{mg}$ spectinomycin/l. All of the germi-

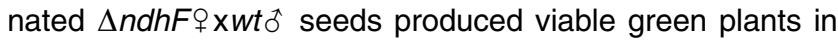
media both in the presence and absence of spectinomycin. All of the germinated $w t+x \Delta n d h F \hat{\sigma}$ seeds (52 out of 58 sown) in spectinomycin-containing media produced white seedlings that did not grow beyond the two-cotyledon stage (Figure 5a) and died soon after. All germinated $w t ? \times \Delta n d h F \hat{\sigma}$ seeds produced green viable plants when sown in media without spectinomycin (Figure 5a). Clearly, the resistance to spectinomycin was always female-inherited and there was no copy of a functional aadA gene in the $\Delta n d h F$ tobacco nucleus.

Plastid genotypes of $10 \mathrm{~F} 1$ individuals of each $w t+\times \Delta n d h F \hat{\delta}$ and $\Delta n d h F \propto \times w t \hat{\delta}$ crosses were assessed by the PCR amplification products with F2 and F4 primers (see 

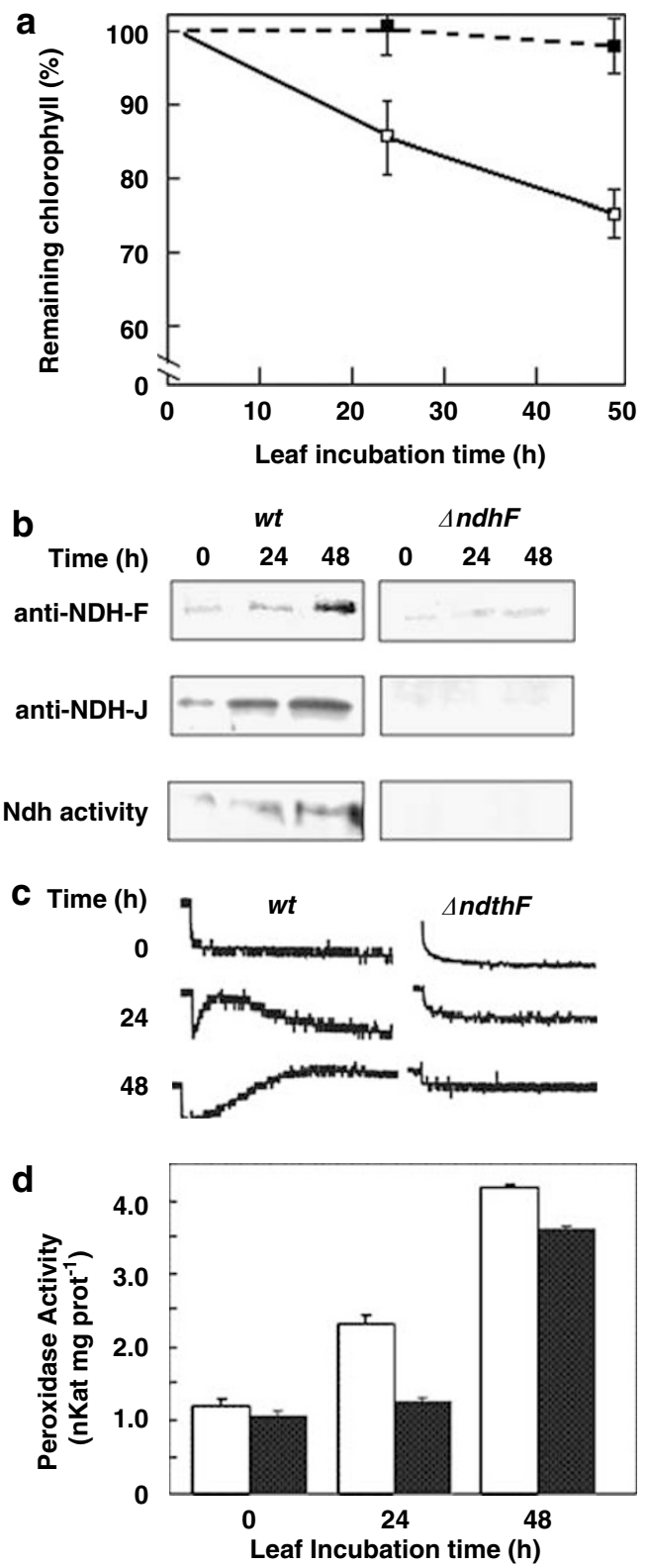

Figure 3 Retention of chlorophyll and evolution of activities in incubated discs of $w t$ and $\Delta n d h F$ tobacco plants. Leaf discs $(1.2 \mathrm{~cm}$ diameter) from the fourth leaves of 70-day-old plants were incubated in water in the dark at $23^{\circ} \mathrm{C}$ for the indicated times. (a) Remaining chlorophyll (\%) in $1 \mathrm{~g}$ disc incubations of wt (- $\square-$-) and $\Delta n d h F(--)$. Values are means \pm S.E. of, at least, five independent assays. Initial values: $1303 \pm 32$ and $1409 \pm 70 \mu \mathrm{g}$ chl/leaf $\mathrm{g}$ in $w t$ and $\Delta n d h F$, respectively. (b) Representative Western blots with NDH-F and NDH-J antibodies and zymograms of the Ndh activity of extracts from wt and $\Delta n d h F$ incubated leaf discs (5 g). Experiments repeated at least three times did not show significant differences. (c) Chlorophyll a fluorescence of incubated leaf discs after interruption of actinic illumination. Experiments repeated at least five times did not show significant differences. (d) Thylakoid peroxidase activity of incubated discs $(5 \mathrm{~g})$ of $w t$ (white bars) and $\Delta n d h F$ (dark bars). Values are means \pm S.E. of, at least, five independent assays

Figure 1b). As Figure 4b shows for two individuals of each cross, the $1928 \mathrm{bp}$ amplified band was always obtained in descendants of $\Delta n d h F+\times w t \hat{\delta}$ crosses, never from $w t+\times \Delta n d h F \hat{o}$ crosses, indicating again that no copy of the
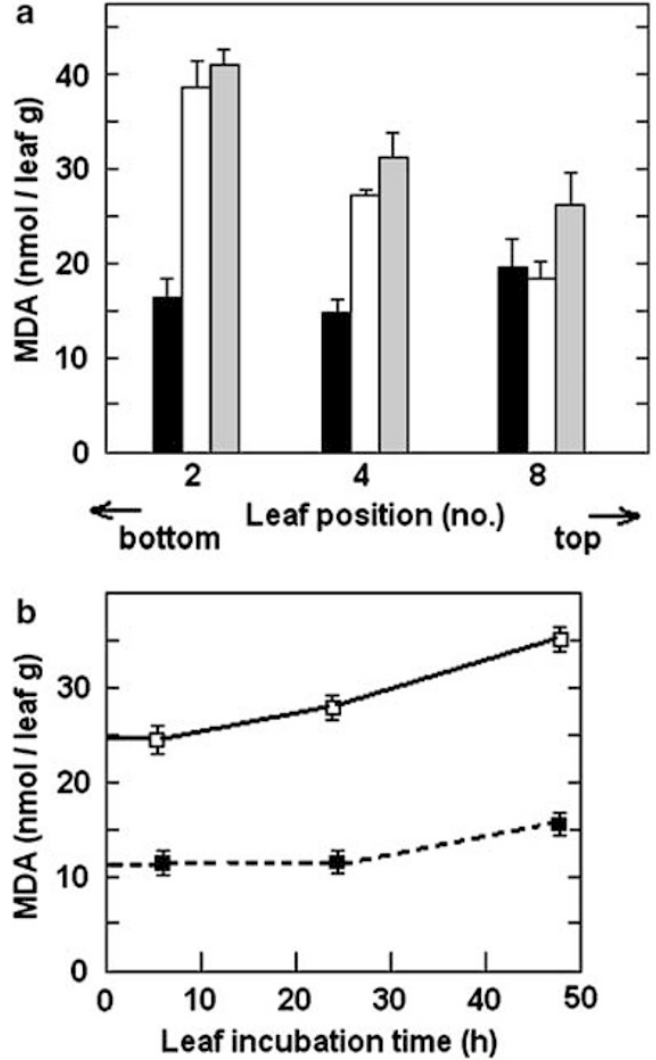

Figure 4 Levels of MDA measured in intact and incubated leaves of wt and $\Delta$ ndhF tobacco plants. (a) MDA was determined in $0.3 \mathrm{~g}$ samples of the second, fourth and eighth leaves of $\Delta n d h F$ (flowering, dark bars) and wt (before flowering, white bars) 72-day-old plants and of 79-day-old wt (flowering, gray bars). Values are means + S.E. of four independent assays. (b) MDA was determined in $0.3 \mathrm{~g}$ of leaf discs from the fourth leaves of 70-day-old plants (wt: $-\square-; \Delta n d h F:-\square-)$ incubated in water in the dark at $23^{\circ} \mathrm{C}$ for the indicated times. Values are means \pm S.E. of six independent assays

aadA-ndhF construction migrated to the nucleus in $\Delta n d h F$ tobacco. F2/F4 primers only amplified the $515 \mathrm{bp}$ band with DNA of $w t$, EcoRV (a transgenic including the aadA cassette in an intergenic region of plastid DNA) and $w t+x \Delta n d h F \delta$ tobacco plants. The $515 \mathrm{bp}$ band accompanied the 1928 band in all $\Delta n d h F{ }^{\circ} \times t_{t} \hat{0}$ descendants with variable and always low intensity, indicating that they were not homoplastomic. Partial sequencing of the $1928 \mathrm{bp}$ band from $\Delta n d h F$ q xwt 0 (Figure S2 of Online Supplementary Information) confirmed that it was a plastid and not mitochondrial DNA sequence.

A final proof linking the delay of senescence in $\Delta n d h F$ tobacco exclusively to the disruption of the plastid $n d h F$ gene was provided by the inheritance of the delayed-senescence phenotype in $\mathrm{F} 1$ of $\Delta n d h F x w t$ crosses. Eight $\mathrm{F} 1$ of each

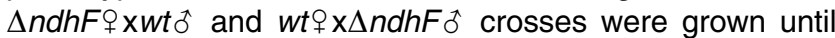
fruit/seed development. The results can be seen in Figure 6: $\Delta n d h F q \times w t$ to descendants showed more than a 30-day delay in senescence with respect to EcoRV and $w t+x \Delta n d h F \hat{\sigma}$ descendants grown simultaneously. Leaf senescence of wt (not shown) and EcoRV controls was simultaneous to $w t$ $\times \Delta$ ndhF $\hat{\sigma} \mathrm{F} 1$ descendants. The absence of the delayed senescence phenotype in EcoRV again indicated that, per se, 
a

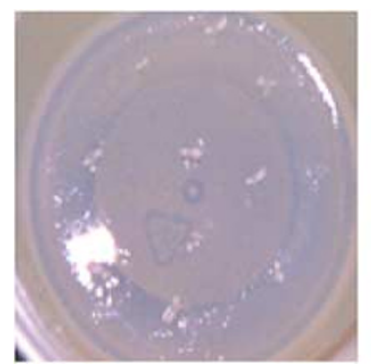

wt $q \times \Delta \boldsymbol{n d} \boldsymbol{h} \boldsymbol{F}$

+ spectinomycin

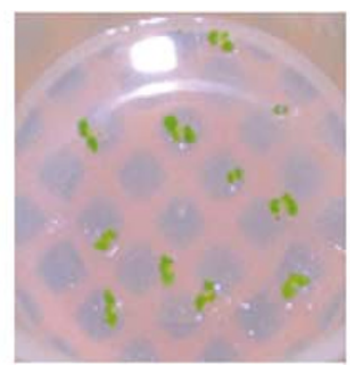

wt $q \times \Delta \boldsymbol{n d h} \boldsymbol{F}_{\diamond}$

- spectinomycin

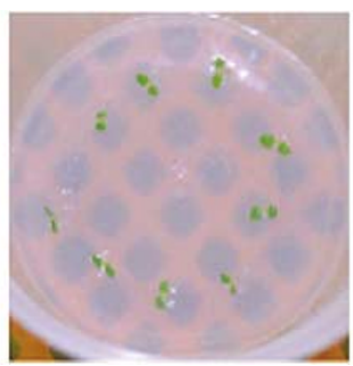

$\Delta n d h \boldsymbol{F} \odot \times$ wt $\curvearrowright$

+ spectinomycin

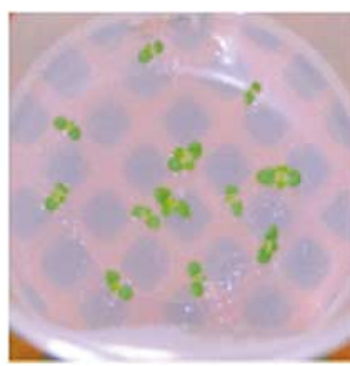

$\Delta n d h \boldsymbol{F} \odot \times$ wt

- spectinomycin

b

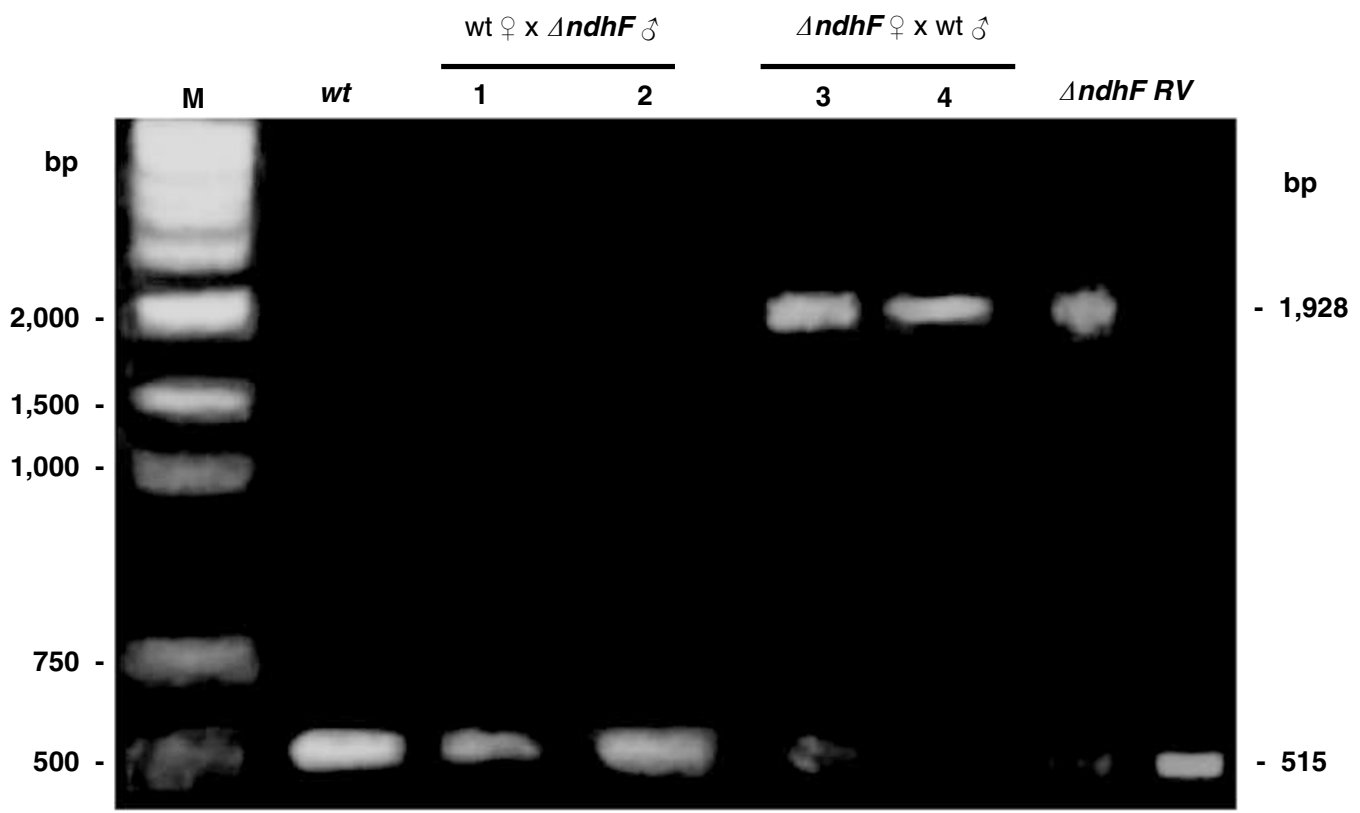

Figure 5 Inheritance of spectinomycin resistance and plastid genotype of $\Delta n d h F$ tobacco. (a) Representative plaques of agar culture media with or without $300 \mathrm{mg}$ spectinomycin/l 15 days after sowing seeds (10-12 per plaque) from the indicated crosses of wt and $\Delta n d h F$ tobaccos. A minimum of six plaques were incubated for each condition and cross. (b) PCR amplification products using primers F2/F4 and DNA isolated from leaves of wt and $\Delta$ ndhF tobacco plants. The plate shows the

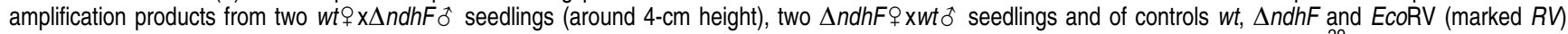
(provided by RM Maier, Munich, Germany) that contained the aadA cassette inserted between the rbcL gene and ORF512 of plastid DNA. ${ }^{29}$ Genotypes of up to 10

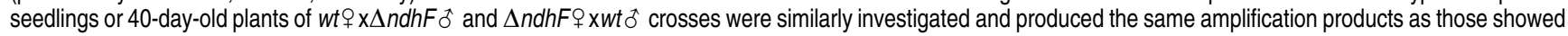
for strains 1,2 and 3,4 , respectively

the aad $A$ gene has no effect on leaf senescence and that the delay of senescence in $\Delta n d h F$ was due exclusively to the functional disruption of the $n d h F$ gene.

\section{Discussion}

In animal PCD, mitochondria integrate signals of proapoptotic and antiapoptotic proteins regulating the release of cytochrome $c$ and the production of ROS that direct subsequent apoptotic processes. ${ }^{8,9,30,31}$ Among other consequences, the release of cytochrome $c$ increases the level of the reduced forms of up-chain transporters that would increase the production of ROS. It seems likely that the increase of the level of complex I (by providing electrons) should also favor the generation of ROS and of cell senescence. The main site for the production of $\cdot \mathrm{O}_{2}^{-}$is complex I and to a lesser extent complexes II and III. ${ }^{31}$ Significantly, the increased transcription of mitochondrial genes for complex I is an early symptom of human aging ${ }^{27}$ and evidence links hereditary complex I deficiencies to longevity. ${ }^{26}$

The Ndh complex of chloroplasts regulates the redox level of cyclic electron transporters by providing electrons that are removed by the Mehler reaction and the coordinated action of SOD and peroxidase when transporters become overreduced. The increase of the level and activity of the Ndh complex during leaf senescence $e^{15,21,23-25}$ strikingly parallels that of the mitochondrial complex I during human aging. ${ }^{27}$ The effects of the two complexes favoring the generation of ROS would indicate that their increases play a key role in PCD. Processes coordinated with complex I/Ndh increase, such as the release of cytochrome $c$ from mitochondria and the decrease of Calvin cycle activity in chloroplasts (both increasing the reduction level of electron transporters and 

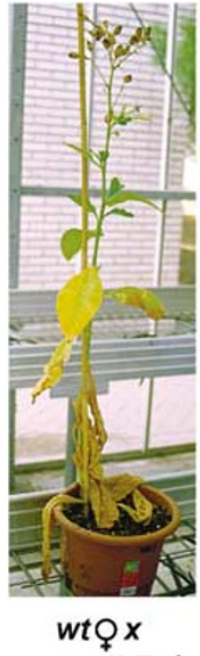

$\Delta n d h F \sigma^{\prime \prime}$
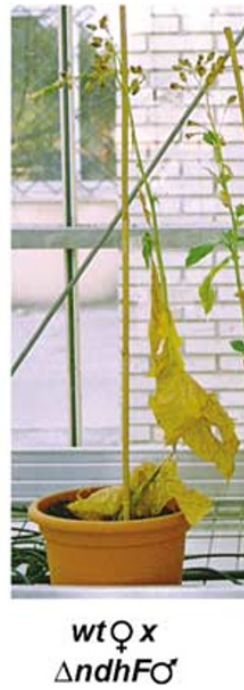

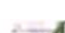

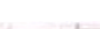
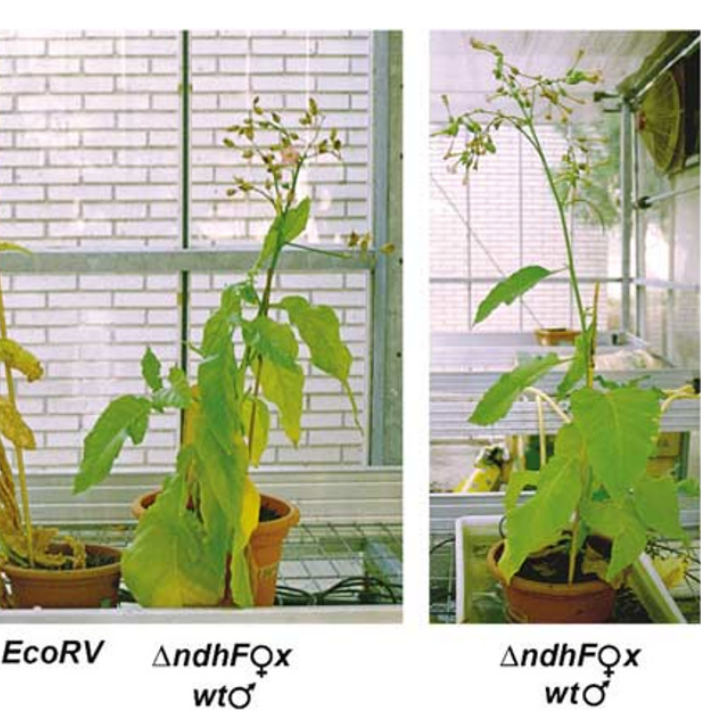

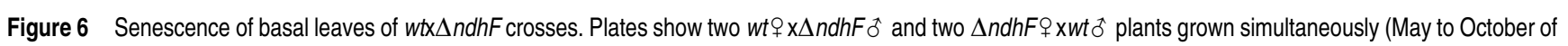
2004) for 135 days in the glasshouse. In the centre: an EcoRV plant grown simultaneously as control

the generation of ROS in the respective organelle) and the decrease of $\operatorname{SOD}^{11,12}$ would contribute to the rise of the steady-state levels of ROS which triggers further PCD processes.

The proposal that the Ndh complex triggers senescence by increasing ROS is strongly supported by the significant higher level of MDA in wt than in $\Delta n d h F$ (Figure 4). On the other hand, paraquat treatment, which increases the production of ROS, enhances senescence symptoms in barley ${ }^{23}$ and tobacco leaves. ${ }^{20}$

It has been proposed that the mitochondrial complex I increases to compensate the energy deficit in aging animal tissues. ${ }^{27}$ In this way, aging tissues would respond like young ones to a lowering of ATP levels. However, in contrast to young tissues, the lowering of ATP levels in aging tissues is not due to its increased demand but to the deterioration of components of the respiratory chain such as cytochrome $c$ oxidase. Therefore, the increase of complex I in aging tissues not only does not restore high rates of ATP production but, what is worse, it increases the reduction level of transporters and the production of ROS, feeding a 'vicious cycle' that leads the cell to death. In chloroplasts, the Ndh complex provides electrons to regulate the redox level of cyclic electron transporters in order to optimize the production of ATP. ${ }^{15}$ The sensing of an oxidative damage threshold and/or a low ATP level would increase the levels of $\mathrm{Ndh}$ in aged leaves. Similarly to mitochondria, the increased activity of the $\mathrm{Ndh}$ complex providing electrons in aged tissues is not compensated because the activity of the Calvin cycle is low and, as there is no increase in chloroplastic SOD, ${ }^{12}$ the generation of $\mathrm{H}_{2} \mathrm{O}_{2}$ is insufficient to remove electrons. Therefore, the chloroplast enters into the autocatalytic cycle of additional - $\mathrm{O}_{2}^{-}$production and oxidative damage and a further reduction of ATP production. According to this hypothesis, the complex I and the Ndh complex play crucial and similar roles in animal and leaf PCD, respectively. The persistence in aged tissues of the mechanisms regulating the levels of complex I and $\mathrm{Ndh}$ complex in mitochondria and chloroplasts, respectively, is not compensated with appropriate changes of the electron removing activities leading the cell to a growing spiral of ROS production and, finally, death. Reactions that feed and remove electrons from transport chains must be finely tuned, and so they are in young healthy cells. Sooner or later, a break of the balance favoring feeding reactions triggers the autocatalytic generation of ROS.

The involvement in leaf senescence is an unexpected role of the plastid ndh genes. Significantly, all tested photosynthetic angiosperms showing developmental-regulated leaf senescence contain plastid ndh genes that are, however, absent in several long-lived-leaf gymnosperms. ${ }^{32}$ The regulation of leaf senescence by chloroplasts opens new lines to investigate whether plastids are targets for factors similar to proapoptotic and antiapoptotic proteins affecting mitochondria in animal PCD. Moreover, the identification of the involvement of $n d h$ genes and chloroplasts in leaf senescence opens ways to control senescence in cultured plants and to delay the deterioration of postharvest vegetables.

\section{Materials and Methods}

Tobacco seeds (Nicotiana tabacum, cv. Petit Havana) (wt, $\Delta n d h F^{20}$ EcoRV (provided by RM Maier, Munich University, Germany), auto- and $w t x \Delta n d h F$ crosses) were aseptically germinated on agar-solidified Murashige/Skoog medium supplemented with $30 \mathrm{~g}$ sucrose/l, at $23^{\circ} \mathrm{C}$ under a 16 -h photoperiod of $100 \mu$ mole photon $/ \mathrm{m}^{2} / \mathrm{s}$ of white light. When indicated, the aseptic culture medium also included $300 \mathrm{mg} / \mathrm{l}$ spectinomycin. Seedlings of about $4 \mathrm{~cm}$ in height were transplanted to compost soil substrate and irrigated with Murashige/Skoog mineral nutrient solution (up to 60 days after germination) and then ( 20 days before flowering) with tap water. After 10 days for adaptation, soil cultures were directly exposed to environment (up to $37^{\circ} \mathrm{C}$ and $2100 / \mu$ photon $/ \mathrm{m}^{2} / \mathrm{s}$ ) or maintained in controlled glasshouse (up to $30^{\circ} \mathrm{C}$ and $200 / \mu$ photon $/ \mathrm{m}^{2} / \mathrm{s}$ ) when indicated.

Total DNA was extracted by the CTAB (cetyltrimethylammonium bromide)-based method. ${ }^{20}$ DNA was amplified according to the standard protocols (one cycle at $94^{\circ} \mathrm{C} 5 \mathrm{~min}$; then $94^{\circ} \mathrm{C}, 30 \mathrm{~s} ; 50^{\circ} \mathrm{C}, 90 \mathrm{~s} ; 72^{\circ} \mathrm{C}$, 
2 min; 30 cycles). The amplified fragments were separated by agarose-gel electrophoresis. PCR products were sequenced in an Applied Biosystems automatic sequencer. The following oligonucleotides were used $\left(5^{\prime}-3^{\prime}\right)$ : C1: TATCCAGCTAAGCGCGAACT; C2: AATTACGTCGCCACCTTCAC; F2: CCCCTTCATGTATGGTTACC; F4: ACCAAAAACAAGCAAGAGGT.

Whole-leaf protein extracts were obtained by homogenization of $1 \mathrm{~g}$ leaves (usually 15-day-old seedlings or fourth leaves of 40 or 70 -day-old plants) with liquid nitrogen in a mortar and suspension in $10 \mathrm{ml}$ of $50 \mathrm{mM}$ potassium phosphate, $\mathrm{pH}$ 7.0, $1 \mathrm{mM}$ L-ascorbic acid, $1 \mathrm{mM}$ EDTA, 1\% polyvinylpyrrolidone and $2 \%$ Triton $\mathrm{X}-100$. The suspensions were gently stirred for $30 \mathrm{~min}$ and then centrifuged at $2000 \mathrm{~g}$ for $30 \mathrm{~min}$. Thylakoid isolation was carried out as described. ${ }^{15}$ Independent thylakoid suspensions were treated with Triton X-100 using a chlorophyll to detergent ratio of $1 / 20$ and $1 / 15(\mathrm{w} / \mathrm{w})$ to solubilize the Ndh complex and plastoquinol peroxidase, respectively. After gently stirring for $30 \mathrm{~min}$, nonsolubilized membranes were separated by centrifugation at $20000 \mathrm{~g}$ for $30 \mathrm{~min}$. All steps were carried out at $4^{\circ} \mathrm{C}$. The absence of significant mitochondrial contamination was regularly confirmed. ${ }^{20}$

Native polyacrylamide gel electrophoresis (PAGE) of solubilized Ndh complex was carried out at $5^{\circ} \mathrm{C}$ in a linear gradient gel of $3-10 \%(\mathrm{w} / \mathrm{v})$ polyacrylamide $(2.5 \%[\mathrm{w} / \mathrm{v}]$ bis-acrylamide) containing $0.1 \%$ Triton $\mathrm{X}-100 .{ }^{23} \mathrm{NADH}$ dehydrogenase zymograms were developed by incubating the gel for $10-50 \mathrm{~min}$ at $30^{\circ} \mathrm{C}$ in darkness with $50 \mathrm{mM}$ potassium phosphate $\mathrm{pH} 8.0,1 \mathrm{mM}$ EDTA, $0.2 \mathrm{mM} \mathrm{NADH}$, and $0.5 \mathrm{mg} / \mathrm{ml}$ nitroblue tetrazolium. No stain developed in the control without NADH. The activity band corresponding to the Ndh complex was identified by immunoblotting. ${ }^{15}$

For immunoblot analyses, leaf crude extracts were subjected to SDSPAGE and transferred to polyvinylidene difluoride (PVDF) membranes (Millipore, Bedford, MA, USA). NDH-F, NDH-J and NDH-K polypeptides were immunodetected with the corresponding rabbit antibodies and the alkaline phosphatase western-blot system (Boehringer, Mannhein, Germany). The NDH-F antibody has been described. ${ }^{25}$ The antibodies against NDH-J and NDH-K were provided by W Koffer and RM Maier (Munich University, Germany).

Chlorophyll fluorescence at room temperature was measured with a portable pulse-modulated fluorometer (PAM-200, Walz, Effeltrich, Germany) as described. ${ }^{21}$ Peroxidase activity of thylakoid solubilized fractions was determined from the rate of increase of absorbance at $250 \mathrm{~nm}$ due to oxidation of $\mathrm{p}$-hydroquinone with $\mathrm{H}_{2} \mathrm{O}_{2}$ as described. ${ }^{20}$ Specific activities are referred to $\mathrm{mg}$ protein. Total protein was determined by the Bradford method using bovine serum albumin as standard. Chlorophyll content was measured by the Arnon method as described. ${ }^{20}$ The level of lipid peroxidation was determined in terms of MDA as described. ${ }^{21}$

\section{Acknowledgements}

This work was supported by grant from the Spanish DGICT. We thank $\mathrm{PH}$ Serrot for kindly suggestions and English corrections.

\section{References}

1. Gan S and Amasino RM (1995) Inhibition of leaf senescence by autoregulated production of cytokinin. Science 270: 1986-1988

2. Noodén LD (2004) Introduction. In Plant Cell Death Processes, Noodén LD (ed) (Amsterdam: Elsevier) pp. 1-18

3. Lam E, Kato $N$ and Lawton M (2001) Programmed cell death, mitochondria and the plant hypersensitive response. Nature 411: 848-853
4. Belenghi B, Salomon M and Levine A (2004) Caspase-like activity in the seedlings of Pisum sativum eliminates weaker shoots during early vegetative development by induction of cell death. J. Exp. Bot. 55: 889-897

5. Yu Z-H, Perdue TD, Heimer YM and Jones AM (2002) Mitochondrial involvement in tracheary element programmed cell death. Cell Death Differ. 9: 189-198

6. Quirino BF, Noh Y-S, Himelblau E and Amasino RM (2000) Molecular aspects of leaf senescence. Trends Plant Sci. 5: 278-282

7. Levine A (1999) Oxidative stress as a regulator of environmental responses in plants. In Plant responses to environmental stress, Lerner HR (ed) (New York: Marcel Dekker, Inc.) pp. 247-264

8. Green DR and Reed JC (1998) Mitochondria and apoptosis. Science 281: 1309-1312

9. Kane DJ, Sarafian TA, Anton R, Hahn H, Gralla EB, Valentine JS, Örd T and Bredesen DE (1993) Bcl-2 inhibition of neural death: decreased generation of reactive oxygen species. Science 262: 1274-1277

10. McRae DG and Thompson JE (1983) Senescence-dependent changes in superoxide-anion production in illuminated chloroplasts from bean leaves. Planta 158: 185-193

11. Orr WC and Sohal RS (1994) Extension of life-span by overexpression of superoxide dismutase and catalase in Drosophila melanogaster. Science 263: $1128-1130$

12. Casano LM, Martín M and Sabater B (1994) Sensitivity of superoxide dismutase transcript levels and activities to oxidative stress is lower in maturesenescent than in young barley leaves. Plant Physiol. 106: 1033-1039

13. Golstein P, Aubry L and Levraud JP (2003) Cell-death alternative model organisms: why and which? Nat. Rev. Mol. Cell Biol. 4: 798-807

14. Sazanov L, Burrows PA and Nixon PJ (1998) The plastid ndh genes code for a NAD-specific dehydrogenase: purification and characterization of a mitochondrial-like complex I from pea thylakoid membranes. Proc. Natl. Acad. Sci. USA 95: 1319-1324

15. Casano LM, Zapata JM, Martín M and Sabater B (2000) Chlororespiration and poising of cyclic electron transport: plastoquinone as an electron transporter between thylakoid NADH dehydrogenase and peroxidase. J. Biol. Chem. 275: 942-948

16. Casano LM, Lascano HR, Martín M and Sabater B (2004) Topology of the plastid Ndh complex and its NDH-F subunit in thylakoid membranes. Biochem. J. 382: $145-155$

17. Sugiura M (1995) The chloroplast genome. Essays Biochem. 300: 49-57

18. del Campo EM, Sabater B and Martín M (2000) Transcripts of the $n d h H-D$ operon of barley plastids: possible role of unedited site III in splicing of the $n d h A$ intron. Nucleic Acids Res. 28: 1092-1098

19. del Campo EM, Sabater B and Martín M (2002) Post-transcriptional control of chloroplast gene expression: accumulation of stable psaC mRNA is due to downstream RNA cleavages in $n d h D$ gene. J. Biol. Chem. 277: 36457-36464

20. Martín M, Casano LM, Zapata JM, Guéra A, del Campo EM, SchmitzLinneweber C, Maier RM and Sabater B (2004) Role of thylakoid Ndh complex and peroxidase in the protection against photo-oxidative stress: fluorescence and enzyme activities in wild-type and ndhF-deficient tobacco. Physiol. Plant. 122: 443-452

21. Guéra A, Calatayud A, Sabater B and Barreno E (2005) Involvement of the thylakoidal $\mathrm{NADH}$-plastoquinone oxidoreductase complex in the early responses to ozone exposure of barley (Hordeum vulgare L.) seedlings. J. Exp. Bot. 56: 205-218

22. Heber U and Walker D (1992) Concerning a dual function of coupled cyclic electron transport in leaves. Plant Physiol. 100: 1621-1626

23. Casano LM, Martín M, Zapata JM and Sabater B (1999) Leaf-age and paraquat concentration-dependent effects on the levels of enzymes protecting against photooxidative stress. Plant Sci. 149: 13-22

24. Martín M, Casano LM and Sabater B (1996) Identification of the product of $n d h A$ gene as a thylakoid protein synthesised in response to photooxidative treatment. Plant Cell Physiol. 37: 293-298

25. Lascano HR, Casano LM, Martín M and Sabater B (2003) The activity of the chloroplastic Ndh complex is regulated by phosphorylation of the NDH-F subunit. Plant Physiol. 132: 256-262

26. Ruiz-Pesini E, Mishmar D, Brandon M, Procaccio V and Wallace DC (2004) Effects of purifying and adaptive selection on regional variation in human mtDNA. Science 303: 223-226 
27. Pich MM, Raule N, Catani L, Fagioli ME, Faenza I, Cocco L and Lenaz G (2004) Increased transcription of mitochondrial genes for complex I in human platelets during ageing. FEBS Lett. 558: 19-22

28. Sabater B and Rodríguez MT (1978) Control of chlorophyll degradation in detached leaves of barley and oat through effect of kinetin on chlorophyllase levels. Physiol. Plant. 43: 274-276

29. Sap $Z$ and Maliga $P$ (1993) High-frequency plastid transformation in tobacco by selection for a chimeric aadA gene. Proc. Natl. Acad. Sci. USA 90: 913-917
30. Ferri KF and Kroemer G (2001) Organelle-specific initiation of cell death pathways. Nat. Cell Biol. 3: E255-E263

31. Dufur $E$ and Larsson N-G (2004) Understanding aging: revealing order out of chaos. Biochim. Biophys. Acta. 1658: 122-132

32. Sabater B, Martín M, Schmitz-Linneweber C and Maier RM (2002) Is clustering of plastid RNA editing sites a consequence of transitory loss of gene function? Implications for past environmental and evolutionary events in plants. Perspect. Plant Ecol. Evol. Systemat. 5: 81-90

Supplementary Information accompanies the paper on Cell Death and Differentiation website (http://www.nature.com/cdd) 